\title{
Synthesis of CLA-rich lysophosphatidylcholine by immobilized MAS1-H108A-catalyzed esterification: effects of the parameters and monitoring of the reaction process
}

\begin{abstract}
Conjugated linoleic acid (CLA)-rich lysophosphatidylcholine (LPC) with many proven beneficial effects is successfully synthesized by an immobilized mutant lipase (MAS1H108A)-catalyzed esterification of glycerophosphorylcholine (GPC) and CLA-rich FAs. Under the optimized conditions (temperature of $55^{\circ} \mathrm{C}$, substrate molar ratio of CLA-rich FAs to GPC of 40:1, and enzyme loading of $300 \mathrm{Ug}-1$ ), LPC content as high as $89.10 \mathrm{~mol} \%$ is achieved, which is exceptionally higher than any ever-reported value. By monitoring the esterification process, it is found that sn1-LPC is easy to synthesize and is the predominant product, while sn2-LPC and phosphatidylcholine (PC) are difficult to synthesize and are with lower content in the final product. The formed sn2-LPC during esterification may mainly attribute to the acyl migration of sn1-LPC and the ratio of sn1-LPC to sn2-LPC finally plateaus at approximately 7. Besides, our results also demonstrate that sn2-LPC is the main template for the synthesis of PC. Finally, a complete reaction scheme for the synthesis of LPC by immobilized MAS1-H108A-catalyzed esterification of GPC and fatty acids is mapped out. Overall, the highest LPC content can be obtained by immobilized MAS1-H108A-catalyzed esterification and there is the first study for systematical studying the reaction process of CLArich LPC synthesis.
\end{abstract}

Keyword: Conjugated linoleic acids; Lysophosphatidylcholine; Glycerophosphorylcholine; Phosphatidylcholine; Reaction processes 